\title{
HER2 specific delivery of methotrexate by dendrimer conjugated anti-HER2 mAb
}

\author{
Rameshwer Shukla ${ }^{1}$, Thommey P Thomas ${ }^{1}$, Ankur M Desai, \\ Alina Kotlyar, Steve J Park and James R Baker Jr \\ Michigan Nanotechnology Institute for Medicine and Biological Sciences, University of \\ Michigan, 9220 MSRB III, Box 0648, Ann Arbor, MI 48109, USA \\ E-mail: rameshwe@umich.edu and jbakerjr@med.umich.edu
}

Received 3 April 2008, in final form 9 May 2008

Published 10 June 2008

Online at stacks.iop.org/Nano/19/295102

\begin{abstract}
Herceptin, a humanized monoclonal antibody that binds to human growth factor receptor-2 (HER2), was covalently attached to a fifth-generation (G5) polyamidoamine dendrimer containing the cytotoxic drug methotrexate. The specific binding and internalization of this conjugate labeled with FITC was clearly demonstrated in cell lines overexpressing HER 2 by flow cytometry as well as confocal microscopic analysis. In addition, binding and uptake of antibody conjugated dendrimers was completely blocked by excess non-conjugated herceptin. The dendrimer conjugate was also shown to inhibit the dihydrofolate reductase with similar activity to methotrexate. Co-localization experiments with lysotracker red indicate that antibody conjugate, although internalized efficiently into cells, has an unusually long residence time in the lysosome. Somewhat lower cytotoxicity of the conjugate in comparison to free methotrexate was attributed to the slow release of methotrexate from the conjugate and its long retention in the lysosomal pocket.
\end{abstract}

\section{Introduction}

Tumor-targeted drug delivery using monoclonal antibodies (mAbs) with specificity towards tumor markers has gained considerable attention in recent years. Targeted drug delivery has the potential to enhance the chemotherapeutic effect while sparing normal tissue from the toxic side effects of systemic administration of these drugs. The drug loading capacity of antibodies can be significantly increased by attaching them to a drug reservoir such as nanoparticles, liposomes or polymers. The advantage of such an approach for cell-specific drug delivery is that higher payloads of the drug can be delivered without compromising the specificity and binding efficacy of the antibody.

Human epidermal growth factor receptor-2 (also known as Neu, ErbB2 or HER2) is a member of the epidermal growth factor receptor subfamily and is considered to be one of the most promising targets in cancer immunotherapy [2]. The HER2 protein has been shown to be overexpressed in a variety of tumors, including breast [14] and ovarian cancers [11], and has been associated with more aggressive tumor growth

1 The first two authors contributed equally to the work. and poorer clinical outcomes [14]. The overexpression of HER2 receptors in tumor cells as well as the presence of an extracellular domain makes it an ideal target for receptor-mediated drug delivery in tumors [2]. Monoclonal antibodies directed against HER2 offer a potential strategy for HER2-targeted delivery. Herceptin is a recombinant humanized monoclonal antibody that is currently in use as a treatment for breast cancer [2]. Antibody-dendrimer conjugates have been previously utilized to target prostatespecific membrane antigen (PSMA) [12], CD antigen [16], delivery of boron [20, 21] and tumor imaging applications. There has been growing interest in the synthesis of herceptin conjugates for applications in gene delivery [1], tumor targeting [18, 15, 7] and imaging applications [3, 6]. In our previous work we reported the synthesis of a dendrimeranti-HER2 mAb conjugate that internalized into the HER2overexpressing cells faster than the antibody [13]. This suggested that a herceptin-targeted dendrimer conjugate has the potential to deliver therapy or imaging agents to the cell interior better than direct antibody conjugates.

In this present study we report the synthesis of antiHER2-targeted PAMAM dendrimer-methotrexate conjugate. Methotrexate (MTX) is an antimetabolite and antifolate drug 
used in the treatment of cancer and autoimmune diseases by inhibiting the metabolism of folic acid. This conjugate was characterized by ${ }^{1} \mathrm{H}$ NMR, HPLC and PAGE. We also studied its binding, internalization in HER2-overexpressing tumor cells by flow cytometry and confocal microscopy. Preincubation experiments with excess antibody prevented the binding and uptake of the conjugate, indicating that the uptake was receptor-mediated. The biological function of the drug was analyzed by DHFR inhibition and XTT assays, respectively. The conjugate was shown to specifically deliver methotrexate to HER2-expressing cell lines as well as retain its antifolate activity. To the best of our knowledge this the first example of the synthesis of HER2-targeted dendrimerdrug conjugate for potential application in tumor-targeted drug delivery.

\section{Experimental section}

\subsection{General}

G5-PAMAM dendrimer was prepared at the Michigan Nanotechnology Institute for Medicine and Biological Sciences, University of Michigan, and analyzed extensively by ${ }^{1} \mathrm{H}$ and ${ }^{13} \mathrm{C}$ NMR, matrix-assisted laser desorption ionization timeof-flight (MALDI-TOF) mass spectrometry, high-performance liquid chromatography (HPLC), gel permeation chromatography (GPC) and polyacrylamide gel electrophoresis (PAGE). The average number of terminal amine groups was determined to be 110 by potentiometric titrations as described previously [10]. The heterobifunctional cross-linkers, sulfosuccinimidyl 3-(2-pyridyldithio) propionate (sulfo-LC-SPDP) and sulfosuccinimidyl (4-iodoacetyl)aminobenzoate (sulfoSIAB), were obtained from Pierce (Rockford, IL). Pre-packed Sephadex G-25 PD-10 columns were purchased from Amersham Pharmacia Biotech (Piscataway, NJ) and equilibrated with degassed eluting buffer before sample introduction. The MCA207 control and MCA207-HER2 breast cancer cell lines were provided by Kevin McDonough (University of Kentucky). Trypsin-EDTA, Dulbecco's phosphate-buffered saline (PBS) and Dulbeccos Modified Eagles Medium (DMEM) were obtained from Gibco/BRL (Gaithersburg, MD). Recombinant dihydrofolate reductase and all other reagents were obtained from Sigma-Aldrich (St Louis, MO). Reversed phase ion-pairing high-performance liquid chromatography was performed using a Waters Delta 600 HPLC system, equipped with a photodiode array detector (Waters 2996) and an autosampler (Waters 717plus). A Phenomenex (Torrance, CA) Jupiter $5 \mu \mathrm{C} 5$ silica-based HPLC column $(250 \mathrm{~mm} \times 4.6 \mathrm{~mm}$, $300 \AA$ ) was used for separation of analytes. The mobile phase for elution of dendrimer conjugates was a linear gradient beginning with 100:0 $\mathrm{H}_{2} \mathrm{O}$ /acetonitrile (CAN) at a flow rate of $1 \mathrm{ml} \mathrm{min}^{-1}$, reaching 20:80 after $30 \mathrm{~min}$. TFA at $0.14 \%$ concentration was added as a counter-ion to water as well as acetonitrile.

\subsection{Synthesis of G5-Ac, 1}

G5 amine dendrimer $(0.265 \mathrm{~g}, 0.0099 \mathrm{mmol})$ and triethyl amine $(0.088 \mathrm{~g}, 0.8635 \mathrm{mmol})$ were dissolved in $30 \mathrm{~mL}$ anhydrous $\mathrm{MeOH}$ and allowed to stir for $30 \mathrm{~min}$. A solution of acetic anhydride $(0.076 \mathrm{~g}, 0.744 \mathrm{mmol})$ in anhydrous $\mathrm{MeOH}$ $(15 \mathrm{ml})$ was added dropwise while stirring. The reaction mixture was allowed to stir overnight at room temperature. After evaporation of the solvent, the residue was dissolved in $\mathrm{H}_{2} \mathrm{O}$ and dialyzed in $10000 \mathrm{MWCO}$ regenerated cellulose dialysis bags initially against PBS buffer followed by water. The partially acetylated dendrimer was lyophilized to give a colorless powder $(0.270 \mathrm{~g}, 91.2 \%)$. The average number of acetyl groups (80) has been determined based on a ${ }^{1} \mathrm{H}$ NMR calibration curve drawn by plotting a ratio of acetyl protons and the sum of all methylene protons versus degree of acetylation [8].

\subsection{Synthesis of G5-Ac-SIAB, 2}

To a solution of G5-Ac, $1(0.042 \mathrm{~g}, 0.0014 \mathrm{mmol})$ in $10 \mathrm{~mL}$ DMSO was added Sulfo-SIAB (0.0080 g, $0.0015 \mathrm{mmol})$ and allowed to stir for $12 \mathrm{~h}$. The unreacted reagents and byproducts were separated by ultrafiltration using a $10 \mathrm{~K}$ MWCO Pelicon device washing initially with PBS and then with DI water. The conjugate was lyophilized to give $0.036 \mathrm{~g}, 85.7 \%$ of conjugate as white powder.

\subsection{Synthesis of G5-Ac-SIAB-Fl, 3}

Fluorescein isothiocyanate $(0.003 \mathrm{~g}, 0.0046 \mathrm{mmol})$ dissolved in DMSO was added to a solution of dendrimer-SIAB conjugate $(0.030 \mathrm{~g}, 0.00096 \mathrm{mmol})$ in DMSO (10 ml) while stirring. The solution was allowed to stir for $18 \mathrm{~h}$ at room temperature. Concentration of the reaction mixture by membrane filtration and further purification on a G-25 Sephadex column gave the dendrimer conjugate which was further purified by membrane filtration and lyophilized to give an orange powder $(0.027 \mathrm{~g}, 90.0 \%)$.

\subsection{Synthesis of G5-Ac-SIAB-Fl-OH, 4}

$0.033 \mathrm{~g}\left(1.04 \times 10^{-6} \mathrm{~mol}\right)$ of $\mathbf{3}$ was reacted with $5.0 \mu \mathrm{l}$ $\left(6.74 \times 10^{-5} \mathrm{~mol}\right)$ of glycidol in $150 \mathrm{ml}$ of DI water. The reaction mixture was stirred vigorously for $6 \mathrm{~h}$ at room temperature. After intensive dialysis in DI water for two days and lyophilization, the yield of the G5-Ac-FITC-OH product was $0.029 \mathrm{~g}$.

\subsection{Synthesis of G5-Ac-SIAB-Fl-O-MTX, 5}

$0.0032 \mathrm{~g}$ of MTX $\left(7.04 \times 10^{-6} \mathrm{~mol}\right)$ was allowed to react with $0.003 \mathrm{~g}\left(1.56 \times 10^{-5} \mathrm{~mol}\right)$ of EDC in $27 \mathrm{ml}$ of DMF and $9 \mathrm{ml}$ of DMSO for $1 \mathrm{~h}$ at room temperature with vigorous stirring. This solution was added dropwise to $150 \mathrm{ml}$ of DI water solution containing $0.0254 \mathrm{~g}\left(7.26 \times 10^{-7} \mathrm{~mol}\right)$ of G5Ac-FITC-OH, 4. The reaction mixture was vigorously stirred for three days at room temperature. After intense dialysis in DI water and lyophilization, the yield of the targeted molecule, G5-Ac-FITC-OH-MTX, was $0.022 \mathrm{~g}$. Further purification was accomplished by membrane filtration (using PBS buffer and DI water). 


\subsection{Synthesis of G5-Fl-HN-MTX, 9}

A protected thiol group was introduced into herceptin, 6 $(0.002 \mathrm{~g}, 0.00013 \mathrm{mmol})$ by reacting with sulfo-SPDP $(0.0006 \mathrm{~g}, 0.00136 \mathrm{mmol})$ at room temperature for $2 \mathrm{~h}$ to give modified antibody, 7. The excess reagent was removed by gel filtration on a Sephadex G-25 column. This conjugate, 7, was then concentrated on a microcon YM100 and immediately reacted with DTT $(0.004 \mathrm{~g}, 0.00013 \mathrm{mmol})$ for $2 \mathrm{~h}$ at room temperature under nitrogen. The removal of excess reagents was performed by gel filtration under a constant stream of nitrogen to give thiolated herceptin, 8 . The thiolated herceptin was reacted with dendrimer conjugate, 5 (0.004 g, $1.11 \times$ $10^{-4} \mathrm{mmol}$ ) in PBS buffer ( $\mathrm{pH} 7.4$ ) to give the final conjugate, 9. The final conjugate (G5-Fl-HN-MTX, 9) was purified by ultrafiltration MWCO 100000 . The dendrimer-antibody conjugate was analyzed by HPLC and UV-vis spectroscopy.

\subsection{Cell culture and in vitro microscopy experiments}

The MCA207 cell lines were cultured in Dulbecco's Modified Eagle Medium (DMEM) supplemented with $10 \%$ fetal calf serum (FCS), 100 units/ml penicillin and $100 \mu \mathrm{g} \mathrm{ml}^{-1}$ streptomycin. Cells were allowed to grow in a monolayer in tissue culture flasks incubated at $37^{\circ} \mathrm{C}$ in a humidified atmosphere containing $5 \% \mathrm{CO}_{2}$ and $95 \%$ air. Cells plated in 24-well plates (for flow cytometry), in $35 \mathrm{~mm}$ glass bottom dishes (for confocal microscopy) or in 96-well plates (for XTT assay), were treated with the conjugate under specified incubation conditions.

The FITC-fluorescence was quantified on a BeckmanCoulter EPICS-XL MCL flow cytometer, and the data were analyzed using Expo32 software (Beckman-Coulter, Miami, FL). The viable cells were gated and the mean FL1fluorescence of 10000 cells was quantified. For confocal microscopy experiments, cells were seeded at a density of $5 \times 10^{5}$ cells/plate on glass bottom culture dishes (Mattek, Ashland, MA) two days prior to the experiment. Cells were incubated with the conjugate in serum-free medium under the specified conditions and analyzed using an Olympus FluoView 500 laser scanning confocal microscope. FITC fluorescence was excited with a $488 \mathrm{~nm}$ blue argon laser and emission was measured through a 505-525 nm barrier filter. Samples were scanned on an Olympus IX-71 inverted microscope using a $60 \times$ water-immersion objective and magnified 2.5 times with FluoView version 4.3 software.

\subsection{Cytotoxicity assay}

For cytotoxicity experiments, cells were seeded in 96well microtiter plates (3000 cells/well) in dialyzed serumcontaining medium. The cells were treated with different concentrations of conjugates for five days with a change in medium and the conjugates on the third day. A colorimetric XTT assay, Roche Molecular Biochemicals (Indianapolis, IN), was performed following the vendor's protocol. After incubation with an XTT labeling mixture, microtiter plates were read on an ELISA reader (Synergy HT, BioTek) at $492 \mathrm{~nm}$ with the reference wavelength at $690 \mathrm{~nm}$.

\subsection{Dihydrofolate reductase assay}

The effect of the G5-FI-HN-MTX on the activity of dihydrofolate reductase (DHFR) was determined using purified DHFR [17]. The reaction mixture containing $50 \mathrm{mM}$ Tris $\mathrm{HCl}$ buffer $\mathrm{pH} 7.5,150 \mathrm{mM} \mathrm{KCl}, 10 \mathrm{mM}$ mercaptoethanol, $120 \mu \mathrm{M}$ NADPH, $100 \mu \mathrm{M}$ dihydrofolate and $0.5 \mu \mathrm{M}$ DHFR. The reaction was started by the addition of the enzyme and the kinetics of reduction in absorbance at $340 \mathrm{~nm}$ was followed in a spectrofluorimeter at room temperature. The rate of change of absorbance was converted to molar concentrations of NADPH using a measured absorbance of 0.75 for a $100 \mu \mathrm{M}$ NADPH solution.

\section{Results}

\subsection{Conjugate synthesis and characterization}

In order to improve targeting efficacy and reduce the nonspecific interactions, an amine-terminated G5 dendrimer was initially partially surface modified with acetic anhydride (75 molar equivalents) in the presence of triethylamine as base (scheme 1). The degree of acetylation was measured by comparing the ratio of $\mathrm{NHCOCH}_{3}$ protons with the sum of all methylene protons in the dendrimer to a calibration curve as described previously [8]. In order to introduce a thiol reactive iodo group on the dendrimer, the heterobifunctional cross-linking agent sulfo-SIAB was conjugated to the partially acetylated dendrimer (G5-Ac, 1) to provide a G5-Ac-SIAB, 2. Compound 2 was reacted with fluorescein isothiocyanate (FITC, 5 molar excess) to give FITC labeled conjugate 3 . The conjugation of glycidol to the acetylated bifunctional device was a necessary preliminary step in order to attach MTX to the dendrimer via an ester linkage and to eliminate the remaining $\mathrm{NH}_{2}$ groups to avoid any non-specific interaction. Conjugation of glycidol to the G5-Ac-SIAB-Fl, 3 converted all the remaining primary amino groups to hydroxyl groups, producing G5-Ac-SIAB-Fl-OH, 4. Methotrexate conjugation via an ester linkage was performed for improved cleavage as opposed to an amide linkage. The methotrexate was conjugated using EDC as coupling agent as previously described $[10,5]$. The number of conjugated methotrexate was calculated to be $\sim 3$ per dendrimer.

A protected thiol group was introduced on the antibody, 6, with water-soluble sulfo-SPDP using standard protocols, and the resultant conjugate, 7 , was purified by gel filtration. The reduction of disulfide bonds on antibody conjugate, 7, was carried out using dithiothreatol, DTT in PBS-EDTA buffer. The resultant thiolated antibody, 8, was carefully purified under an inert atmosphere in degassed PBS-EDTA buffer. To minimize the free antibody, tenfold molar excess of dendrimer conjugate was used in the antibody-dendrimer coupling reaction (scheme 2); the unreacted thiols were quenched with $N$-ethylmaleimide to minimize the dimer formation due to cross-linking of dendrimer to antibodies. Free unreacted dendrimer was removed by filtration with a $100 \mathrm{~K}$ MWCO microcon. The resultant conjugate, 9, was analyzed using PAGE, which shows a band above the antibody band that confirms the conjugation and an absence of a band for the free 


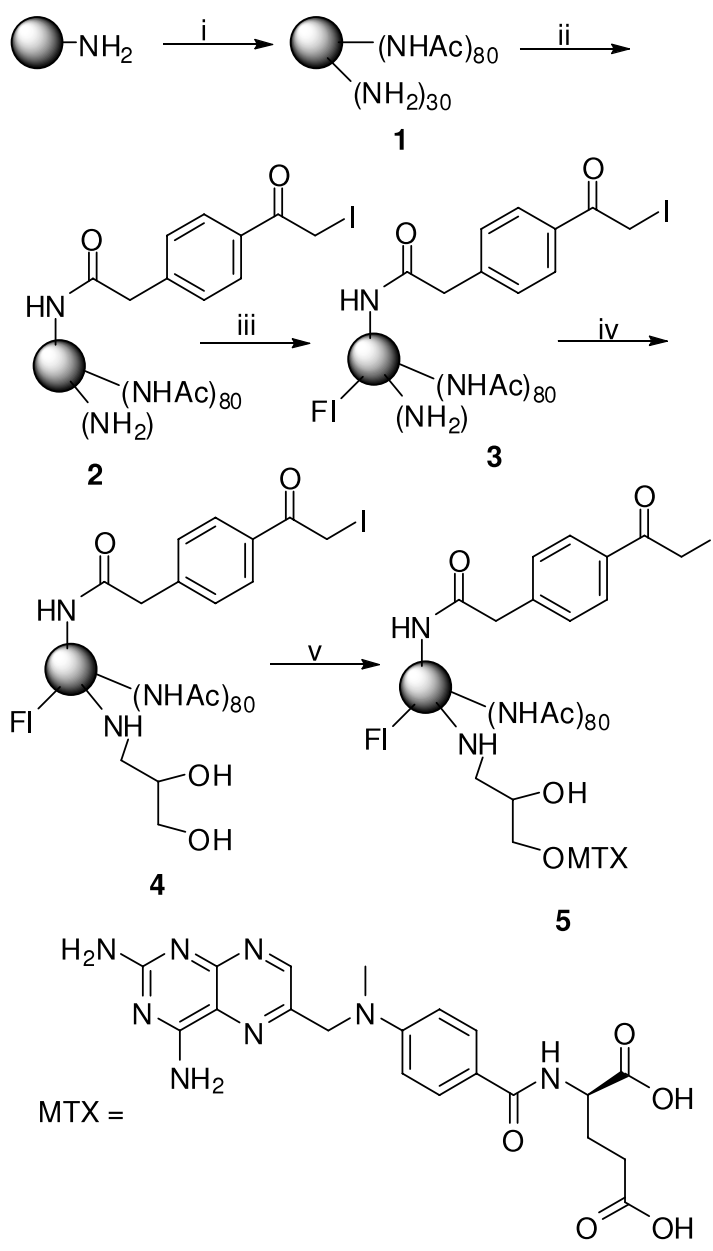

\section{i) $\mathrm{Ac}_{2} \mathrm{O}, \mathrm{Et}_{3} \mathrm{~N}$ ii) Sulfo-SIAB iii) FITC iv) Glycidol v) $M T X, E D C$}

Scheme 1. Surface modification of the dendrimer

dendrimer. The fluorescence of the conjugate gives an indirect proof of conjugation as the fluorescent label was attached to the dendrimer. The HPLC analysis of the final conjugate shows a homogeneous peak, which indicates that, under the present reaction conditions, there was minimum cross-linking which appears to be due to the steric hindrance posed by the relatively larger size of the antibody.

\subsection{In vitro biological studies of G5-FI-HN-MTX}

The G5-Fl-HN-MTX conjugate bound to the HER2-expressing MCA207 cell line in a dose-dependent fashion, whereas it failed to bind to the control MCA207 cells lacking the receptor. The control dendrimer G5-Fl without the targeting $\mathrm{HN}$ molecule did not bind to either cell line (figure 1). The confocal microscopic analysis showed the internalization of the conjugate into the cytosolic compartment (figure 2) in the same way as previously reported [13].

The cytotoxic potential of the G5-Fl-HN-MTX conjugate was tested in HER2-expressing cells by XTT assay. As shown in figure 3, when HER2-overexpressing MCA 207 cells were incubated with $1 \mu \mathrm{M}$ G5-Fl-HN-MTX conjugate

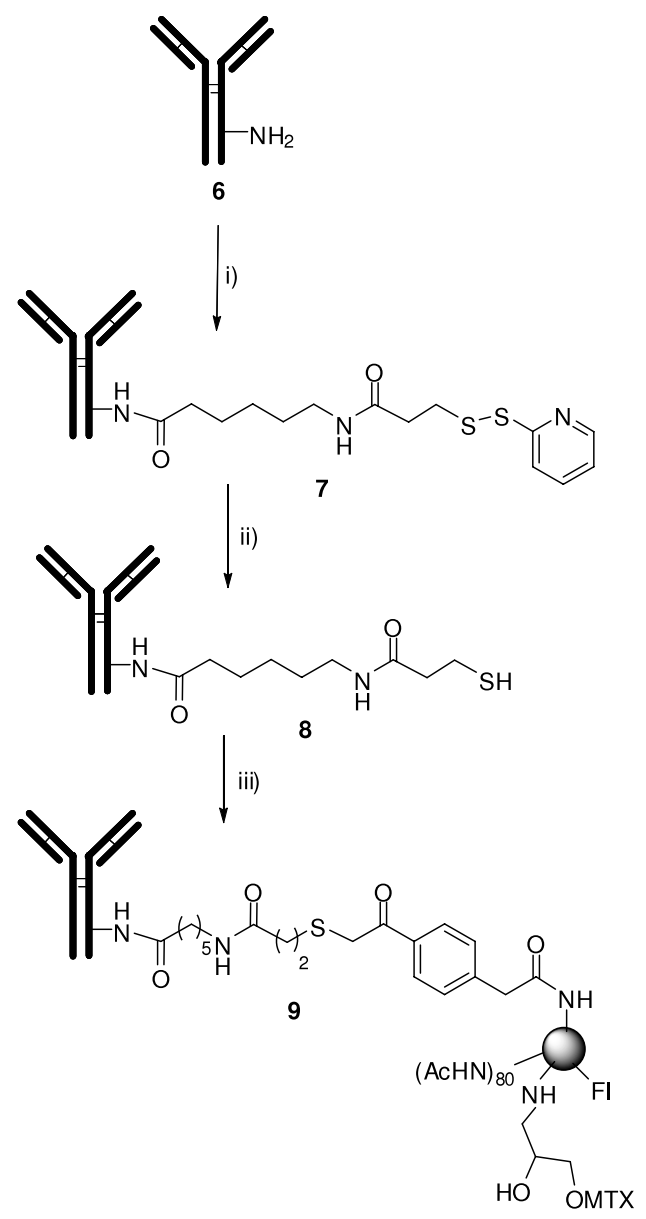

i) Sulfo-SPDP ii) DTT iii) 5

Scheme 2. Conjugation of the dendrimer to anti-HER2 mAb.

a decrease of $25 \%$ in cell growth was observed. However, under similar conditions herceptin or the control conjugate G5-Fl, which does not contain either drug or targeting group, were not growth inhibitory up to a concentration of $3 \mu \mathrm{M}$, whereas free MTX was significantly cytotoxic at $100 \mathrm{nM}$. We also tested the effect of this conjugate on the activity of the enzyme DHFR. The dendrimer-MTX conjugate inhibited purified DHFR in the same way as methotrexate whereas free herceptin did not have any effect on the activity of DHFR independently or on the inhibition of the enzyme by free MTX (figure 4). The inhibition of purified DHFR demonstrates that the methotrexate conjugated to the dendrimer was biologically functional. Similar results were obtained when we used the MCA-HER2 cell cytosol as the source of DHFR (figure 5). We performed a co-localization experiment using lysotracker red and the targeted conjugate G5-Fl-HN-MTX. As shown in figure 6 the conjugate was co-localized with the lysotracker dye for $48 \mathrm{~h}$. This indicates that the targeted conjugate is effectively taken up in the cells and is localized in the lysosomal compartment.

\section{Discussion}

The major disadvantage of most antitumor drugs is their high non-specificity, leading to toxic side effects. Successful drug 


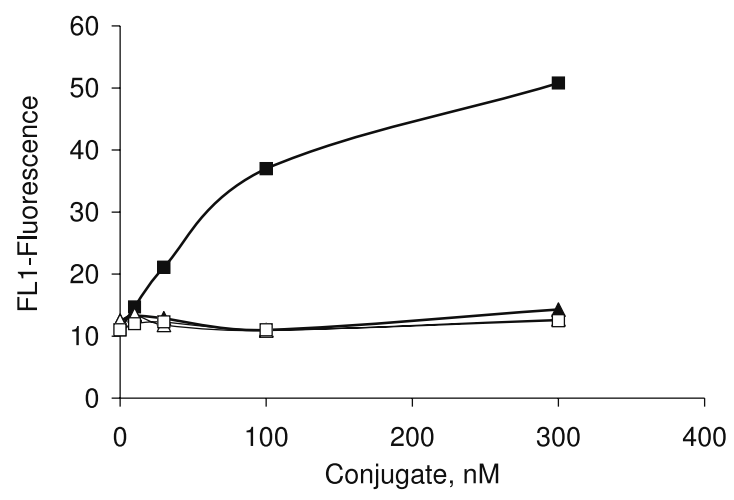

Figure 1. Dose-dependent binding of G5-FI-HN-MTX (filled symbols) and the control conjugate G5-FI (open symbols) in HER2-positive (square symbols) and control (triangle symbols) MCA207 cells. Cells were incubated with different concentrations of the conjugates G5-FI at $37^{\circ} \mathrm{C}$ for $1 \mathrm{~h}$, and the mean

FL1-fluorescence was determined in a flow cytometer.
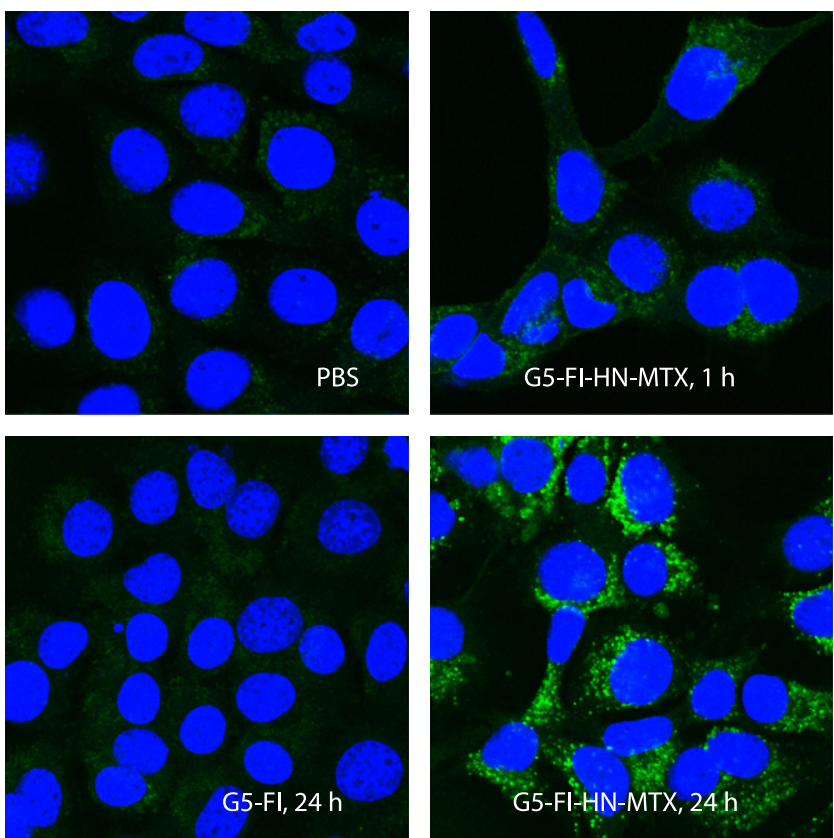

Figure 2. Internalization of G5-FI-HN-MTX in MCA207-HER2 cells. Cells grown on coverslips were incubated with $300 \mathrm{nM}$ each of G5-FI-HN-MTX or the control conjugate G5-FI at $37^{\circ} \mathrm{C}$ for 1 and $24 \mathrm{~h}$. Cells were rinsed, fixed with paraformaldehyde, mounted using a solution containing the nuclei stain DAPI and the fluorescence measured in an Olympus confocal microscope. The green and the blue stains show FI-fluorescence and nuclei stained with DAPI, respectively.

targeting has the potential to lower the dosage of a drug required for a therapeutic response while limiting systemic toxicity. Antibodies have been explored as drug carrier systems, but attaching drugs to them often results in the loss of antibody binding efficacy or problems with the aqueous solubility of the immunoconjugates. These problems can be circumvented by attaching a water-soluble drug reservoir to the antibodies. The G5-PAMAM dendrimer is stable, nonimmunogenic and contains, on average, 110-128 primary amines on the surface that provide ample reactive sites for

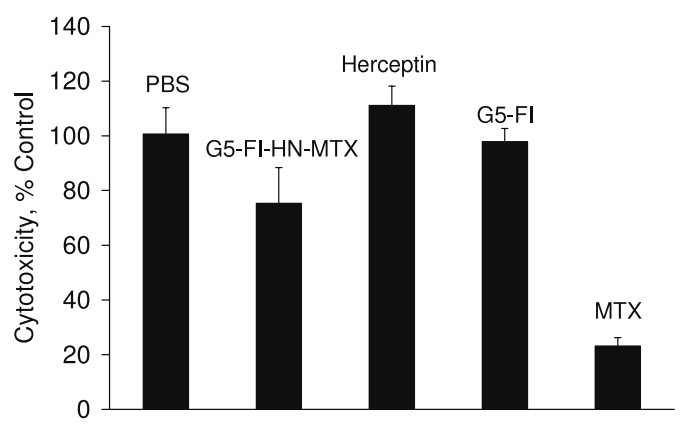

Figure 3. Effect of G5-FI-HN-MTX on cell growth. MCA-HER2 cells were incubated with $1 \mu \mathrm{m}$ of each of G5-FI-HN-MTX, G5-Fl and free herceptin, or $100 \mathrm{~nm}$ of methotrexate (MTX) for five days with a change in medium plus the conjugates after two days. The cell proliferation was determined by XTT assay as described in section 2 .

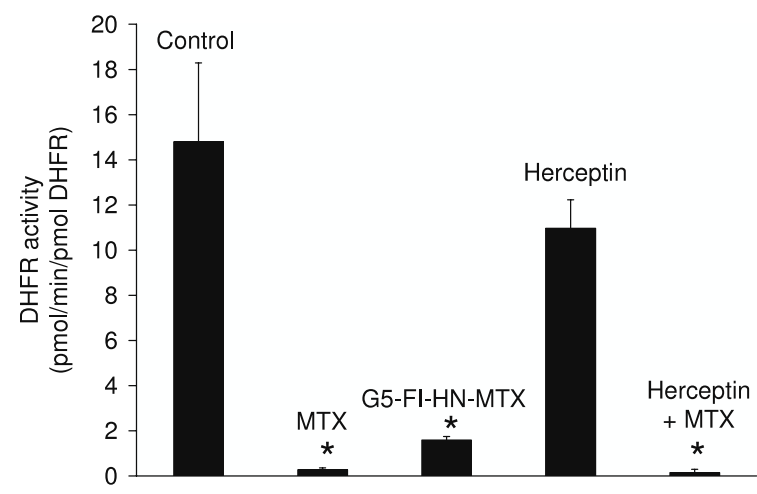

Figure 4. Inhibition of DHFR activity by G5-FI-HN-MTX. The effect of the compounds shown $(0.75 \mu \mathrm{M})$ on the activity of recombinant DHFR was tested as described in section 2. $* p<0.05$ versus control.

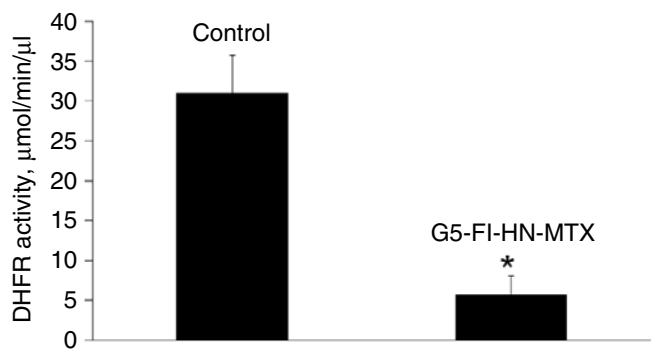

Figure 5. DHFR activity in MCA-HER2 cell homogenate. Cell homogenate was prepared and the activity of DHFR was determined in the absence or presence of G5-FI-HN-MTX $(0.75 \mu \mathrm{M})$ as described in section $2 . * p<0.05$ versus control.

conjugation for the development of complex drug delivery systems [5]. The overexpression of HER2 protein in a high percentage of epithelial tumors should permit the selective targeting of tumor cells with anti-HER2 mAb. Therefore, in the present study we synthesized a conjugate of $(\mathrm{HN})$ with a PAMAM dendrimer conjugated to methotrexate as the cytotoxic drug. The amine terminated G5 dendrimer was partially surface acetylated in order to minimize the non-specific charge interactions and aggregate formation. 
B
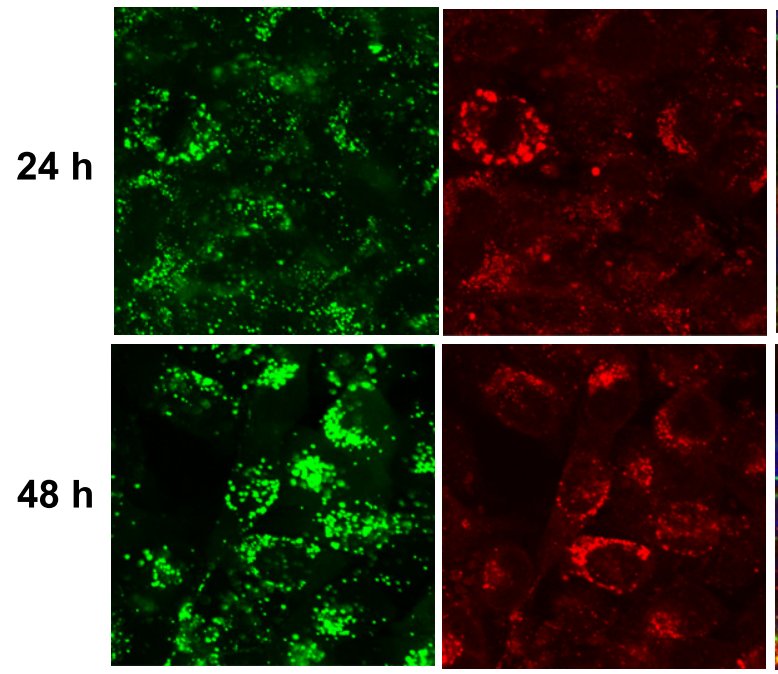

C
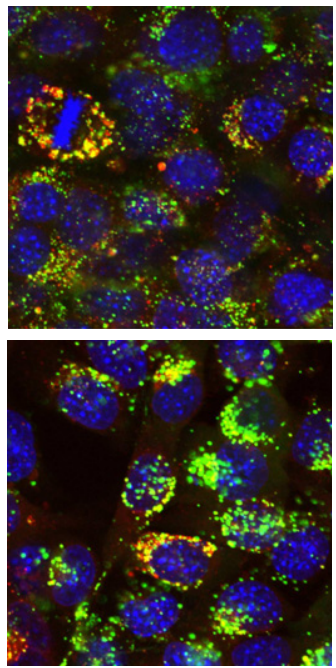

Figure 6. Co-localization of G5-FI-HN-MTX and lysotracker red in MCA207-HER2 cells. Cells grown on cover slips were incubated with $300 \mathrm{nM}$ each of G5-Fl-HN-MTX or the control conjugate G5-Fl at $37^{\circ} \mathrm{C}$ for 24 and $48 \mathrm{~h}$. Cells were stained with lysotracker red, rinsed, fixed with paraformaldehyde, mounted using solution containing the nuclei stain DAPI and fluorescence measured in an Olympus confocal microscope as given in section 2. The green, red and blue stains show FI-fluorescence (panel A) and lysotracker red (panel B), respectively, for the same viewing area. Panel C shows an overlay of Panel A and B along with cell nuclei stained with DAPI.

The partially acetylated dendrimer was reacted with a heterobifunctional linker, sulfo-SIAB, to provide a thiol reactive iodoacetamido group on the surface of the dendrimer. The number of iodo linkers attached to the dendrimer was calculated to be $\sim 4$ linkers per dendrimer based on ${ }^{1} \mathrm{H}$ NMR integral values. In order to detect the conjugate by flow cytometry or for visual imaging via confocal microscopy a fluorescent probe is required. We labeled the partially acetylated dendrimer with fluorescein isothiocyanate (FITC) as the fluorescent imaging moiety. G5-Ac-SIAB was reacted with 5 molar equivalents of FITC dissolved in dimethyl sulfoxide (DMSO). After purification, the conjugate was characterized using ${ }^{1} \mathrm{H}$ NMR spectroscopy and high-performance liquid chromatography (HPLC). The ${ }^{1} \mathrm{H}$ NMR of the conjugate showed broad signals in the aromatic region for the FITC as well as the phenyl ring of the SIAB linker. The number of dye molecules attached to the dendrimer was calculated to be $\sim 4$ based on UV/vis spectroscopy and ${ }^{1} \mathrm{H}$ NMR. For methotrexate conjugation we chose to use an ester linker for improved cleavage by cellular esterases [9]. However, our prior work has shown that intracellular release of the drug was not necessary for the biological activity of methotrexate. To support this, we incubated folic-acid-targeted dendrimermethotrexate conjugate in PBS buffer as well as folic acid receptor expressing $\mathrm{KB}$ cell extract and did not observe any appreciable release of methotrexate. This indicates that the methotrexate conjugate possibly imparts its cytotoxic activity as a methotrexate-dendrimer conjugate. This result is also supported by the recent results of Gurdag et al showing cytotoxity of methotrexate-dendrimer conjugates prepared via an amide bond that is very less likely to be hydrolyzed [4]. We modified the remaining primary amino groups of the dendrimer with glycidol to give a hydroxyl surface. Methotrexate was conjugated to the hydroxyl surface dendrimer G5-Ac-SIABFl-OH, 4 using the carbodiimide coupling reaction. The
anti-HER2 mAb was modified with protected thiol linker which, upon reduction with DTT gave thiolated antibody, 7. The thiolated antibody was reacted with G5-Ac-SIAB-FlMTX to give thioether-linked HER2 mAb-targeted dendrimerMTX conjugate. The remaining thiol groups were capped with N-ethyl maleimide to prevent cross-linking. The G5FI-HN-MTX conjugate was shown to bind HER2-expressing MCA207 cell line in a dose-dependent fashion, whereas it does not to bind to the control MCA207 cells. Also the control dendrimer conjugate $\mathrm{G} 5-\mathrm{Fl}$ without the targeting $\mathrm{HN}$ molecule does not show any binding to both cell lines (figure 2). From the confocal microscopic analysis internalization of the conjugate into the cytosolic compartment was observed in the same way as previously reported [13].

The conjugate showed $25 \%$ inhibition of cell growth in HER2-overexpressing MCA 207 cell lines as measured by XTT assay whereas the herceptin and the control conjugate did not show any growth inhibition. However, under similar conditions G5-FA-MTX conjugate shows $\sim 75 \%$ inhibition of cell growth in folic acid receptor overexpressing cell lines. Reduced cytotoxicity of cetuximab-targeted dendrimermethotrexate bioconjugates was also reported recently by $\mathrm{Wu}$ et al [19]. They hypothesized that conjugation of a mAb might alter the binding affinity of methotrexate with dihydrofolate reductase, leading to the loss of its antifolate activity. Thus, we tested the effect of this conjugate on the activity of the enzyme DHFR to ascertain whether the antibody is indeed posing any steric hindrance on methotrexate's ability to bind and inhibit the enzyme DHFR. The conjugate was shown to inhibit purified DHFR as well as DHFR obtained from MCA 207 HER 2 cell homogenate, whereas herceptin alone did not have any effect on the activity of DHFR independently or on the inhibition of the enzyme by free MTX. This data indicates that the reduced cytotoxicity is not a result of lowered affinity 
of the conjugate towards enzyme DHFR and the antifolate activity of the methotrexate is retained in the conjugate. Next, we analyzed the intracellular localization of the dendrimerdrug conjugate. Our co-localization experiments indicate that the conjugate localized with lysotracker red, a marker for late endosomes and lysosomes, within $1 \mathrm{~h}$. However, the most striking feature was the unusually long residence time (48 h) of the conjugate in the lysosomal pocket, which might explain the reduced cytotoxicity as the steric hindrance posed by the antibody or dendrimers does not allow intracellular esterases to release the drug and the conjugate as a whole also does not get an opportunity to bind the DHFR because of extended retention in the lysosomes. We are currently in the process of conjugating a lysosome-disrupting agent to the dendrimerantibody conjugate to facilitate faster release of the conjugate from lysosomes.

\section{Conclusions}

In summary, we have synthesized and characterized a HER2targeted dendrimer-methotrexate conjugate which provides a general approach for the synthesis of herceptin-targeted dendrimer-drug conjugates. The conjugate selectively targets HER2-overexpressing cell lines in vitro and is able to maintain its antifolate activity. This principle could be applied to the delivery of either one or a combination of drugs to enhance their therapeutic index or to imaging agents to improve their diagnostic potential. The reduced cytotoxicity of this conjugate can be possibly attributed to the slow release of the drug and long retention of the conjugate in lysosomes. Although the conjugate was less toxic to cells than methotrexate alone in vitro, however, it is possible that this bioconjugate might be superior for in vivo application due to its reduced toxicity for HER2-overexpressing breast cancers resulting from specific targeting of tumor cells. We are currently working on testing the therapeutic efficacy of this conjugate in animal tumor models.

\section{Acknowledgments}

The authors gratefully acknowledge financial support from the National Cancer Institute contract N01-CM-97065-32, National Institutes of Health 1 R01 CA 119409, Karlene Kulp Endowed Research Fund of the University of Michigan Comprehensive Cancer Center and Michigan Economic Development Corporation-Life Sciences Corridor Fund, under award GR-472.

\section{References}

[1] Chiu S H, Ueno N T and Lee R J 2004 Tumor-targeted gene delivery via anti-HER2 antibody (trastuzumab, Herceptin (R)) conjugated polyethylemmine J. Control. Release 97 357-69

[2] Cho H S, Mason K, Ramyar K X, Stanley A M, Gabelli S B, Denney D W and Leahy D J 2003 Structure of the extracellular region of HER2 alone and in complex with the Herceptin Fab Nature 421 756-60

[3] Copland J A, Eghtedari M, Popov V L, Kotov N, Mamedova N, Motamedi M and Oraevsky A A 2004 Bioconjugated gold nanoparticles as a molecular based contrast agent: implications for imaging of deep tumors using optoacoustic tomography Mol. Imaging Biol. 6 341-9

[4] Gurdag S, Khandare J, Stapels S, Matherly L H and Kannan R M 2006 Activity of dendrimer-methotrexate conjugates on methotrexate-sensitive and -resistant cell lines Bioconjug. Chem. 17 275-83

[5] Kukowska-Latallo J F, Candido K A, Cao Z Y, Nigavekar S S, Majoros I J, Thomas T P, Balogh L P, Khan M K and Baker J R 2005 Nanoparticle targeting of anticancer drug improves therapeutic response in animal model of human epithelial cancer Cancer Res. 65 5317-24

[6] Liu J, Li J, Rosol T J, Pan X L and Voorhees J L 2007 Biodegradable nanoparticles for targeted ultrasound imaging of breast cancer cells in vitro Phys. Med. Biol. 52 4739-47

[7] Liu X, Wang Y, Nakamura K, Kubo A and Hnatowich D J 2008 Cell studies of a three-component antisense MORF/tat/Herceptin nanoparticle designed for improved tumor delivery Cancer Gene Ther. 15 126-32

[8] Majoros I J, Keszler B, Woehler S, Bull T and Baker J R 2003 Acetylation of poly(amidoamine) dendrimers Macromolecules $\mathbf{3 6}$ 5526-9

[9] Majoros I J, Myc A, Thomas T, Mehta C B and Baker J R 2006 PAMAM dendrimer-based multifunctional conjugate for cancer therapy: synthesis, characterization, and functionality Biomacromolecules 7 572-9

[10] Majoros I J, Thomas T P, Mehta C B and Baker J R 2005 Poly(amidoamine) dendrimer-based multifunctional engineered nanodevice for cancer therapy J. Med. Chem. 48 5892-9

[11] Olayioye M A, Neve R M, Lane H A and Hynes N E 2000 The ErbB signaling network: receptor heterodimerization in development and cancer Embo J. 19 3159-67

[12] Patri A K, Myc A, Beals J, Thomas T P, Bander N H and Baker J R 2004 Synthesis and in vitro testing of J591 antibody-dendrimer conjugates for targeted prostate cancer therapy Bioconjug. Chem. 15 1174-81

[13] Shukla R, Thomas T P, Peters J L, Desai A M, Kukowska-Latallo J, Patri A K, Kotlyar A and Baker J R 2006 HER2 specific tumor targeting with dendrimer conjugated anti-HER2 mAb Bioconjug. Chem. 17 1109-15

[14] Slamon D J, Clark G M, Wong S G, Levin W J, Ullrich A and McGuire W L 1987 Human-breast cancer-correlation of relapse and survival with amplification of the Her-2 Neu Oncogene Science 235 177-82

[15] Sun B, Ranganathan B and Feng S S 2008 Multifunctional poly(D,L-lactide-co-glycolide)/montmorillonite (PLGA/MMT) nanoparticles decorated by Trastuzumab for targeted chemotherapy of breast cancer Biomaterials 29 $475-86$

[16] Thomas T P et al 2004 Detection and analysis of tumor fluorescence using a two-photon optical fiber probe Biophys. J. 86 3959-65

[17] Thompson K, Vinh D B N and McIvor R S 1992 Assay for expression of methotrexate-resistant dihydrofolate-reductase activity in the presence of drug-sensitive enzyme J. Pharmacol. Toxicol. Methods 28 167-73

[18] Wartlick H, Michaelis K, Balthasar S, Strebhardt K, Kreuter J and Langer K 2004 Highly specific HER2-mediated cellular uptake of antibody-modified nanoparticles in tumour cells J. Drug Target. 12 461-71

[19] Wu G, Barth R F, Yang W L, Kawabata S, Zhang L W and Green-Church K 2006 Targeted delivery of methotrexate to epidermal growth factor receptor-positive brain tumors by means of cetuximab (IMC-C225) dendrimer bioconjugates Mol. Cancer Ther. 5 52-9

[20] Yang W L et al 2006 Molecular targeting and treatment of EGFRvIII-positive gliomas using boronated monoclonal antibody L8A4 Clin. Cancer Res. 12 3792-802

[21] Yang W L et al 2008 Molecular targeting and treatment of composite EGFR and EGFRvIII-positive gliomas using boronated monoclonal antibodies Clin. Cancer Res. 14 883-91 